\title{
Asymptotic Equivariant Index of Toeplitz Operators on the Sphere
}

\author{
This article is dedicated to M. Sato, whose work has always been
}

a great source of inspiration

by

Louis Boutet De Monvel

\begin{abstract}
We illustrate the equivariant asymptotic index described in [6, 8, in the case of spheres $\mathbb{S}^{2 N-1} \subset \mathbb{C}^{N}$, equipped with a unitary action of a compact group, for which this theory is more explicit. The article is mostly a review article, except for the last section ( $\S 5$ ) in which we describe conjecturally some very natural generators of the relevant K-theory for a torus action on a sphere, generalizing in our Toeplitz operator context the generators proposed by M. F. Atiyah [2] for the transversally elliptic pseudodifferential theory.
\end{abstract}

2010 Mathematics Subject Classification: 19L47, 32A25, 53D10, 58J20, 58J40.

Keywords: Toeplitz operators, index, equivariant K-theory, contact manifolds.

\section{$\S 1$. Toeplitz operators on the sphere}

Let $X=\mathbb{S}^{2 N-1}$ denote the unit sphere, bounding the unit ball $\mathbb{B}$ in $\mathbb{C}^{N}$.

We will denote by $\mathbb{H}$ (or $\mathbb{H}_{X}$ ) the kernel of the tangent Cauchy-Riemann system $\bar{\partial}_{b}$, i.e. the space of boundary values of holomorphic functions on $\mathbb{B}$ that are of moderate growth near the boundary (these are distributions on $X$ ).

We denote by $S$ (or $S_{X}$ ) the $S z e g \ddot{o}$ projector, i.e. the orthogonal projector of $L^{2}(X)$ on $\mathbb{H} \cap L^{2}$ :

$$
S f(z)=\int_{X} S(z, \bar{w}) f(w) d \sigma(w) \quad \text { with } \quad S(z, \bar{w})=\frac{1}{v}(1-z \cdot \bar{w})^{-N}
$$

where $v=2 \pi^{N} /(N-1)$ ! is the volume of $X$.

This is a contribution to the special issue "The golden jubilee of algebraic analysis".

Communicated by M. Kashiwara. Received December 20, 2009.

L. Boutet de Monvel: UPMC Paris 6, UMR 7586, F-75005 Paris, France;

e-mail: boutet@math.jussieu.fr

(C) 2011 Research Institute for Mathematical Sciences, Kyoto University. All rights reserved. 
$S$ is a Fourier integral operator (FIO) with complex phase (cf. [9]); it acts continuously on all Sobolev spaces $H^{s}(X)$ with range $\mathbb{H}^{s}=\mathbb{H} \cap H^{s}(X)$.

A Toeplitz operator of degree $m$ is an operator of the form $f \in \mathbb{H} \mapsto T_{P}(f)=$ $S(P(f))$ where $P$ is a pseudodifferential operator $(\psi \mathrm{DO})$ of degree $m$ on $X$; it is continuous $\mathbb{H}^{s} \rightarrow \mathbb{H}^{s-m}$ for all $s$. In particular if $\phi$ is a smooth function on $X$, it defines a Toeplitz operator $T_{\phi}(f \mapsto S(\phi f))$. The operators initially introduced by Toeplitz are the $T_{\phi}$ on the circle $(N=1)$.

Toeplitz operators give rise to a symbolic calculus identical to the pseudodifferential calculus: the sphere $X$ is a contact manifold with contact form induced by $\lambda=\operatorname{Im}(\bar{z} \cdot d z)$. The associated symplectic cone is the set $\Sigma \subset T^{\bullet} X$ of positive multiples of $\lambda^{1}$ this is one half of the line bundle, the set of real characteristics of $\bar{\partial}_{b}$, which carries the microsingularities of functions in $\mathbb{H}$ or of $S$. The symbol $\sigma_{m}(A)$ of a Toeplitz operator $A$ of degree $\leq m$ is a homogeneous function on $\Sigma$ of degree $m$, the restriction to $\Sigma$ of the symbol of $P$ if $A=T_{P}$, and we have

$$
\sigma_{m+m^{\prime}}(P \circ Q)=\sigma_{m}(P) \sigma_{m^{\prime}}(Q), \quad \sigma_{m+m^{\prime}-1}([P, Q])=\frac{1}{i}\left\{\sigma_{m}(P), \sigma_{m^{\prime}}(Q)\right\} .
$$

Modulo operators of degree $-\infty$, Toeplitz operators are local; they define a sheaf of algebras on $X$, denoted $\mu \mathbb{H}$, acting on the sheaf of holomorphic microfunctions ( $\operatorname{ker} \bar{\partial}_{b} \bmod C^{\infty}$ ); this is locally isomorphic to the sheaf of pseudodifferential operators $\left(\bmod C^{\infty}\right)$ in $N$ real variables acting on microfunctions, on the cosphere $S^{*} \mathbb{R}^{N}$.

Example. If $P=P\left(z, \partial_{z}\right)$ is a holomorphic differential operator (defined in a neighborhood of $\mathbb{B}$ ), it obviously acts on $\mathbb{H}$ and defines a Toeplitz operator $T_{P}$ (or $P$ if this does not lead to confusion).

We will use in particular the operator $\rho$, which is an elliptic Toeplitz operator of degree 1:

$$
\rho=z \cdot \partial_{z}=\sum z_{j} \partial_{j}, \quad f=\sum f_{\alpha} z^{\alpha} \mapsto \sum|\alpha| f_{\alpha} z^{\alpha} .
$$

Any Toeplitz operator is equivalent $\left(\bmod C^{\infty}\right)$ to an asymptotic sum: $T_{P} \sim$ $\sum_{m \leq m_{0}} \rho^{m} T_{\phi_{m}}$; the "coefficients" $\phi_{m} \in C^{\infty}(X)$ are smooth functions on $X$ and are uniquely determined ${ }^{2}$

The following relation was pointed out in [6]:

Lemma 1. On the sphere $X=\mathbb{S}^{2 N-1}$ of $\mathbb{C}^{N}$ we have

$$
\partial_{j}=(\rho+N) T_{\bar{z}_{j}} \quad \text { with as above } \quad \rho=\sum_{j=1}^{N} z_{j} \partial_{j} .
$$

\footnotetext{
${ }^{1} T^{\bullet} X$ denotes the cotangent bundle $T^{*} X$ deprived of its zero section.

${ }^{2}$ This is only asymptotic $\left(\bmod C^{\infty}\right)$; the series usually does not converge. This does not really give rise to a practical "total" symbolic calculus.
} 
It is sometimes practical to identify $\Sigma$ with $\mathbb{C}^{N}-\{0\}$, with Liouville form $\lambda=\operatorname{Im}(\bar{z} \cdot d z)$ and symplectic form $\omega=\operatorname{Im}(d \bar{z} \cdot d z)$ (twice the canonical one), the coordinate functions $z_{j}, \bar{z}_{j}$ being homogeneous of degree $\frac{1}{2}$ (however this can lead to confusion: in this identification the symbol of $T_{z_{j}}$, resp. $T_{\partial_{j}}$, is $\frac{1}{|z|} z_{j}$, resp. $\left.|z| \bar{z}_{j}\right)$.

\section{§2. Equivariant index}

Let $G$ be a compact Lie group, with a linear unitary action on $\mathbb{C}^{N}$. Then $G$ preserves holomorphic functions and the Szegö kernel (which is invariant under the action of $U(N)$ ), and we may consider invariant Toeplitz operators.

We denote by $\mathfrak{g}$ the Lie algebra of $G$. Any $\gamma \in \mathfrak{g}$ produces a vector field tangent to the sphere $X$, whose holomorphic part defines a Toeplitz operator of the form

$$
L_{\gamma}=i \sum a_{p q} z_{q} \partial_{p}
$$

where $\left(a_{p q}\right)$ is a hermitian matrix. The symbol of $\frac{1}{i} L_{\gamma}$, when we identify $\Sigma$ with $\mathbb{C}^{N}-\{0\}$ as above, is the hermitian form $q(z)=\sum a_{p q} z_{q} \bar{z}_{p}$ (symbol map moment map).

Definition 2. We denote by char $\mathfrak{g} \subset \Sigma$ the set of points of $\Sigma$ where all symbols $\sigma\left(\frac{1}{i} L_{\gamma}\right)=\left\langle A_{\gamma} z \mid z\right\rangle, \gamma \in \mathfrak{g}$, vanish.

The base $Z \subset X$ is the set of points where all vector fields $L_{\gamma}(\gamma \in \mathfrak{g})$ are orthogonal to the contact form $\lambda$ (this still makes sense on any contact $G$-manifold: if $X$ is the cosphere bundle of a $G$-manifold $M$, we have char $\mathfrak{g}=T_{G}^{*} M$, the set of covectors orthogonal to the orbits of $G$ ).

If $G$ is a torus acting diagonally: $g z=\left(e^{2 i \pi \mu_{k}(\gamma)} z_{k}\right)$ if $g=\gamma \bmod \mathbb{Z}^{n}$ $\left(\mu_{k} \in \mathbb{Z}^{n} \subset \mathfrak{g}^{*}\right), Z$ is the inverse image under the map $z \mapsto\left(\lambda_{k}=\left|z_{k}\right|^{2}\right)$ of the convex rational polyhedron $\left\{\sum \lambda_{k} \mu_{k}=0\right\}$ in $\mathbb{R}_{+}^{N}\left(\lambda_{k} \geq 0, \sum \lambda_{k}=1\right)$.

For index theory it is also necessary to consider Szegö projectors on the space of sections of $G$-vector bundles (for which the definition and existence of equivariant generalized Szegö projectors works as well, cf. [3, 7]). In the case where $X$ is a sphere it is enough to consider "trivial" bundles, of the form $X \times E$, where $E$ is some finite representation of $G\}^{3}$ for these the holomorphic Szegö projector is well defined $\left(S \otimes \operatorname{Id}_{E}\right)$.

If $P$ is an equivariant Toeplitz operator acting on holomorphic sections of $G$-vector bundles, and $P \sim 0$ near char $\mathfrak{g}$, then the $G$-trace $\operatorname{Tr}_{G}(P)$ is defined as

\footnotetext{
${ }^{3}$ Because any $G$-bundle is stably trivial, i.e. there exist trivial bundles $E^{\prime}, F$ such that $E \oplus F \sim E^{\prime}$.
} 
in 2. It is a distribution on $G$; if $P$ is of trace class (i.e. of degree $<-N)$ it is the continuous function $g \mapsto \operatorname{Tr}_{G}(P)(g)=\operatorname{tr}(g \circ P)$. In the general case, let $\Delta$ be a biinvariant elliptic operator of degree $>0$ (e.g. the Casimir operator of some faithful representation), $\Delta_{X}$ the Toeplitz operator it defines on $X$ (which is elliptic outside of char $\mathfrak{g}$ ); dividing repeatedly we get $P=\Delta_{X}^{m} Q+R$ where $R$ is of degree $-\infty$, and for large $m$ the quotient $Q$ is of trace class; we have $\operatorname{Tr}_{G}(P)=$ $\Delta^{m} \operatorname{Tr}_{G}(Q)+\operatorname{Tr}_{G}(R)$. The $G$-trace makes sense for any operator $P \sim 0$ near char $\mathfrak{g}$; its restriction to equivariant operators is a trace, i.e. $\operatorname{Tr}_{G}(A B)=\operatorname{Tr}_{G}(B A)$ if $A, B$ are both equivariant and one of them is $\sim 0$ near char $\mathfrak{g}$.

We will say that $P$ is $G$-ellipti $4^{4}$ if it is elliptic on char $\mathfrak{g}$, i.e. its principal symbol is invertible there (thus also near char $\mathfrak{g}$ ). The definition of M. F. Atiyah [2] can be reproduced:

Definition 3. If $P$ is equivariant and $G$-elliptic, its $G$-index is defined as the virtual representation ker $P$-coker $P$. Its character is the distribution $\operatorname{Tr}_{G}(1-Q P)$ $-\operatorname{Tr}_{G}(1-P Q)$ where $Q$ is any $G$-parametrix of $P$ (i.e. $Q$ is equivariant, $Q P \sim 1$, $P Q \sim 1$ near char $\mathfrak{g})$.

ker $P$ and coker $P$ are both tempered representations of $G$, and their characters are well defined as distributions. The index is the difference of their characters: we have

$$
\operatorname{Ind}_{G}(P)=\sum \frac{I_{\alpha}}{d_{\alpha}} \chi_{\alpha}
$$

where $\alpha$ ranges over the set of irreducible representations, with character $\chi_{\alpha}$ and dimension $d_{\alpha}$, and $I_{\alpha}$ is the index of the restriction of $P$ to the isotypic components of type $\alpha\left(I_{\alpha} / d_{\alpha}\right.$ is integral $)$.

These definitions extend to equivariant complexes of Toeplitz operators.

The symbol of an equivariant $G$-elliptic system $P$ defines a K-theoretical element $[P]^{\text {abs }} \in K_{0}^{G}(\operatorname{char} \mathfrak{g} \cup\{0\})$; the $G$-index of $P$ only depends on this (because it is additive and deformation invariant) ${ }^{5}$

\section{§3. Asymptotic index}

Szegö projectors and Toeplitz operators are defined more generally on any compact oriented contact manifold $X$ (whose associated symplectic cone is again the halfline subcone of $T^{\bullet} X$ consisting of positive multiples of the contact form).

Let us recall from [7, 4, 5, that if $\Sigma$ is a symplectic subcone of $T^{\bullet} M$, with $M$ a smooth compact manifold, a generalized Szegö projector associated to $\Sigma$ is a

\footnotetext{
4 "Transversally elliptic" in 2], but "transversally" is not suitable in the Toeplitz context.

${ }^{5}$ This would in general not make sense for contact manifolds other than spheres.
} 
self-adjoint elliptic Fourier integral projector $S$ of degree $0\left(S=S^{*}=S^{2}\right)$, whose complex canonical relation $\mathcal{C}$ is $\gg 0$, with real part the diagonal $\operatorname{diag} \Sigma$ (elliptic means that the principal symbol of $S$ does not vanish on $\Sigma, \gg 0$ refers to the work of A. Melin and J. Sjöstrand [13, 14]).

Such a projector always exists; its range $\mathbb{H}$ is the associated "Toeplitz space" (following the terminology of [7]). As before, the Toeplitz operators corresponding to $S$ are the operators on $\mathbb{H}$ of the form $u \in \mathbb{H} \mapsto T_{P}(u)=S P S(u)$ with $P$ a pseudodifferential operator on $M$. They form an algebra (i.e. sums and products of Toeplitz operators are Toeplitz operators). Modulo smoothing operators, they form a sheaf acting on $\mu \mathbb{H}$, locally isomorphic to the sheaf of pseudodifferential operators acting on the sheaf of microfunctions in $p$ variables if $\operatorname{dim} \Sigma=2 p$.

The generalized Szegö projectors associated to $\Sigma$ are "essentially unique": if $S, S^{\prime}$ are two generalized Szegö projectors, $S^{\prime}$ induces a Fredholm map $\mathbb{H} \rightarrow \mathbb{H}^{\prime}$. More generally if $u, u^{\prime}$ are two embeddings of $\Sigma$ in cotangent bundles $T^{\bullet} M, T^{\bullet} M^{\prime}$ and $\mathbb{H}, \mathbb{H}^{\prime}$ the corresponding "Toeplitz spaces", there always exists a FIO $F$, whose (complex) canonical relation is $\gg 0$ with real part the graph of $u^{\prime} \circ u^{-1}$, inducing a Fredholm map $\mathbb{H} \rightarrow \mathbb{H}^{\prime}$ ("adapted" in the terminology of [7, or [4]).

The pair $\left(\mathcal{A}_{\Sigma}, \mu \mathbb{H}\right)$ consisting of the sheaf of micro Toeplitz operators (i.e. mod smoothing operators) acting on $\mu \mathbb{H}$ is well defined, up to (non-unique) isomorphism: it only depends on the symplectic cone $\Sigma$, not on the embedding.

All the constructions above allow a compact group action (cf. [7), i.e. $S$ can be constructed invariant; if $u, u^{\prime}$ above are equivariant, $F$ can be constructed equivariant, etc 6

If $X$ is a contact $G$-manifold and we have chosen an invariant Szegö projector, Atiyah's theory of transversally elliptic indices can be reproduced to some extent: the characteristic set char $\mathfrak{g}$ is defined exactly as above, its base $Z$ is again the set of points of $X$ where the infinitesimal generators $L_{\gamma}$ of the action of $G$ are orthogonal to the contact form. Again there is a notion of elliptic system (or complex) of Toeplitz operators (i.e. elliptic along char $\mathfrak{g}$ ), and such a system has a $G$-index, i.e. a trace class virtual representation, whose character is a distribution on $G: 7$

However because from the contact data alone the Szegö projector is at best known up to a compact operator, $\mathbb{H}$ is at best known modulo a finite-dimensional space and there is no hope to compute the index by local or differential formulae

\footnotetext{
${ }^{6}$ In these constructions one uses means repeatedly, and the compactness condition on the group cannot be relaxed.

${ }^{7}$ We recall that a virtual representation is of trace class if its character $\sum m_{\alpha} \chi_{\alpha}$ converges in distribution sense, i.e. the coefficients $m_{\alpha}$ (which are integers) are bounded by some power of the eigenvalues of a faithful Casimir operator (or any biinvariant elliptic operator).
} 
involving the principal symbol alone, without further data. (This is even worse for $G$-vector bundles, even in the case of the sphere if a "trivial" or at least holomorphic structure is not given. There is a pseudodifferential analogue: if $E$ is a vector bundle on a manifold $M$ and $p \in C^{\infty}\left(S^{*} M, L(E)\right)$ a projector defined on the cosphere, there always exists a pseudodifferential projector $P$ with symbol $p$ (compatible with the action of $G$ if there is one), but this is obviously not unique, any two such differ by a compact operator; the projection from the range of one to the other is a Fredholm operator, on the index of which there can be no control from the topological data alone.)

A remedy to this, used in [8, is to introduce the asymptotic $G$-index, which is well defined for equivariant Toeplitz operators, only depends on their symbols, and still encodes pertinent information if $G$ is not finite:

Definition 4. The asymptotic index is the absolute index modulo a finite virtual representation; its character is the singularity (distribution $\bmod C^{\infty}$ ) of the distribution character of the "absolute" $G$-index.

(Note that a series $\sum m_{\alpha} \chi_{\alpha}$ has a $C^{\infty}$ sum iff the sequence of coefficients $m_{\alpha}$ is rapidly decreasing; if the $m_{\alpha}$ are integers it is a finite sum.)

If $u$ is an isomorphism from $\Sigma \subset T^{\bullet} M$ to $\Sigma^{\prime} \subset T^{\bullet} M^{\prime}$ (equivalently a contact isomorphism on the bases), and $F: \mathbb{H} \rightarrow \mathbb{H}^{\prime}$ an adapted FIO as above, it can be used to transport $G$-elliptic systems and preserves the asymptotic $G$-index (not the absolute index).

The asymptotic index of an equivariant $G$-elliptic Toeplitz system $P$ obviously only depends on its top order symbol, which defines an element $[P] \in K^{G}(X-Z)$, where $K^{G}(X-Z)$ is the group of virtual $G$-bundles with compact support in $X-Z$; its elements are stable equivalent classes of equivariant $G$-bundle homomorphisms $E \rightarrow F$ on the sphere, invertible on $Z$ (the base of char $\mathfrak{g}$ ). Since the asymptotic index is also additive and deformation invariant, we get (cf. [6]):

Proposition 5. The asymptotic index of an equivariant $G$-elliptic system $P$ only depends on the K-theoretic element $[P] \in K^{G}(X-Z)$ it defines. The index defines a linear map

$$
\text { asInd }: K^{G}(X-Z) \rightarrow \operatorname{Sing}(G) \text {. }
$$

(The K-theoretic element $[P] \in K^{G}(X-Z)$ is well defined for any contact manifold; in the case of the sphere, it is the image of $[P]^{\text {abs }}$ mentioned above ${ }^{8}$

\footnotetext{
${ }^{8}$ An equivariant map $u: E \rightarrow F$ invertible on $Z$ defines the 0-element iff $u$ can be stably deformed into an invertible homomorphism on the whole sphere $X$, i.e. it lies in the image of the obvious map $K_{0}^{G}(\mathbb{B}) \rightarrow K_{0}^{G}(\operatorname{char} \mathfrak{g})$.
} 


\section{§4. Embedding}

It is possible to transfer the index from a $G$-sphere to a larger one. Let $X=\mathbb{S}^{2 N-1}$ be the unit sphere of $V=\mathbb{C}^{N}$, with a unitary action of $G$, as in $\S 1$. Let $V^{\prime}, V^{\prime \prime}$ be two orthogonal invariant subspaces and $Y=V^{\prime} \cap X$, an invariant subsphere.

Definition 6. We denote by $d_{V^{\prime \prime}}$ (or $d^{\prime \prime}$ ) the partial holomorphic de Rham complex, acting on $z^{\prime \prime}$-differential forms with holomorphic coefficients in all variables $\left(z^{\prime}, z^{\prime \prime}\right)$ :

$$
\left(\mathbb{E}^{k}, d^{\prime \prime}\right) \quad \text { with } \mathbb{E}^{k}=\mathbb{H} \otimes \bigwedge^{k} V^{\prime \prime *}, d^{\prime \prime} \omega\left(z^{\prime}, z^{\prime \prime}\right)=\sum d z_{j}^{\prime \prime} \frac{\partial \omega}{\partial z_{j}^{\prime \prime}}
$$

$\left(V^{\prime \prime *}\right.$ denotes the dual of $V^{\prime \prime}$ ). This is obviously equivariant (if $V^{\prime}, V^{\prime \prime}$ are invariant), and its cohomology is $\mathbb{H}^{\prime}$ ( 0 in positive degree). The symbol of $d^{\prime \prime}$ is, except for the factor $a=\sigma\left(z \cdot \partial_{z}\right)>0$, the exterior multiplication by $\sum \bar{z}_{j} d z_{j}$.

Formula (1.2) shows that the symbol of $d^{\prime \prime}$ is, up to a positive factor $(\sigma(\rho))$, adjoint to the symbol of the Koszul complex $k_{V^{\prime \prime}}$, which is the complex (in negative degrees)

$\left(\mathbb{E}_{-k}, I_{z^{\prime \prime}}\right) \quad$ with $I_{z^{\prime \prime}}$ the interior product $\left.\omega\left(z^{\prime}, z^{\prime \prime}\right) \mapsto \sum z_{j}^{\prime \prime} \partial_{z_{j}^{\prime \prime}}\right\lrcorner \omega$.

The K-theoretical element $\left[k_{V^{\prime}}\right]=\left[d_{V^{\prime \prime}}\right] \in K_{Y}^{G}(X)$ is precisely the Bott element, defining the Bott isomorphism $E \mapsto E \otimes\left[k_{V^{\prime}}\right]$ of $K^{G}(Y)$ to $K_{Y}^{G}(X)$ (the corresponding assignment of operators is $\left.P \mapsto P \otimes d_{V^{\prime \prime}}\right)$.

Thus this "obvious" transfer takes a $G$-elliptic system $P$ on $Y$ to a $G$-elliptic system $P^{\prime}$ on $X$ which has the same asymptotic $G$-index, and whose associated K-theoretical element $\left[P^{\prime}\right]$ is the Bott image of that of $P 9^{9}$

More generally, let $Y, X$ be two compact contact $G$-manifolds and $u: Y \rightarrow X$ an invariant contact embedding (i.e. the contact form $\lambda_{Y}$ is a positive multiple of $u^{*} \lambda_{X}$, equivalently $u$ is the base map of a symplectic embedding). Let $Y^{\prime}$ be the image submanifold, with symplectic cones $\Sigma_{Y} \subset \Sigma_{X}$ and $G$-invariant Toeplitz spaces $\mathbb{H}_{Y} \subset \mathbb{H}_{X}$ (e.g. $\Sigma_{Y}$ is realized as a symplectic subbundle of $T^{\bullet} M, Y=S^{*} M$, $\left.\mathbb{H}_{X}=L^{2}(M)\right)$.

Then (cf. [8]) $\mathbb{H}_{Y}$ is the set of solutions of a complex $D_{Y \rightarrow X}$ of Toeplitz operators mimicking $\bar{\partial}_{b}$.

This can be used to transfer the computation of the asymptotic on $Y$ to that on $X$. The K-theoretic element $\left[k_{Y \rightarrow X}\right] \in K_{Y}^{G}(X)$ defined by the symbol of $D_{Y \rightarrow X}$

\footnotetext{
${ }^{9}$ In the case of spheres, there is also a result for the absolute index, provided we work with trivial bundles, or at least with holomorphic bundles, so that the range of the Szegö projector is unambiguously defined; the K-theoretical element $[P]$ is then an element of $K^{G}(\operatorname{char} \mathfrak{g})$, the $\mathrm{K}$-theory with compact support of the cone char $\mathfrak{g}$ (including the origin), rather than $K^{G}(X-Z)$.
} 
is precisely the element which defines the Bott isomorphism $K^{G}(Y) \rightarrow K_{Y}^{G}(X)$. If a K-theoretical element $u \in K^{G}(X-Z)$ is supported by $Y$, it belongs to the range of the canonical homomorphism $K_{Y}^{G}(X-Z) \rightarrow K^{G}(X-Z)$ : it is the image via the Bott homomorphism of an element of $K^{G}(X-Z)$ and the corresponding Toeplitz system has the same equivariant index.

Since any compact contact $G$-manifold can be embedded in a contact sphere with a linear unitary action of $G$, this theoretically reduces the index problem to the problem on the sphere, essentially studied by M. F. Atiyah [2] (at least when $G$ is a torus with a symmetric action as indicated below).

\section{§5. Generators}

If $V_{0} \subset V$ is the fixed subspace of $G$, it is immediate that any $G$-elliptic system can be deformed equivariantly into a $G$-elliptic system also elliptic outside of the orthogonal complement of $V_{0}$, whose K-theoretical element is then supported by $X \cap V_{0}^{\perp}$. Thus for the computation of the index, we are reduced to the case where $V$ has no trivial component.

Definition 7. We will say that the action of $G$ is elliptic if char $\mathfrak{g}$ is empty ${ }^{10}$ (the base $Z$ is empty).

For the cotangent sphere of a $G$-manifold $M$, this would just mean that $M$ is a finite union of open orbits; for a sphere or a contact manifold, it is less trivial.

If the action of $G$ is elliptic, any equivariant bundle homomorphism $u: E \rightarrow F$ is $G$-elliptic; its $G$-index is $\operatorname{Ind}_{G} E-\operatorname{Ind}_{G} F$ (where for short we have denoted by $\operatorname{Ind}_{G} E$ the $G$-index of the zero map $E \rightarrow 0$ ).

From now on we will suppose that the base is a sphere $X \subset V$ as in $\$ 1$ and that $G$ has no fixed point. As mentioned above any $G$-bundle on the sphere is stably isomorphic to a trivial (product) bundle. If the action of $G$ is elliptic, any equivariant bundle homomorphism is $G$-elliptic. The index of the trivial bundle of rank 1, with space of holomorphic sections $\mathbb{H}_{V}$, is the polynomial algebra:

$$
\operatorname{Ind}_{G}([1])=\sum S^{n} V^{* n}\left(=\operatorname{Ind}_{G}\left(\mathbb{H}_{V}\right)\right) .
$$

The $G$-index of the trivial bundle $X \times E$ is $E \otimes \operatorname{Ind}\left(\mathbb{H}_{V}\right)$.

We now further suppose that $G$ is a torus $\mathbb{R}^{n} / \mathbb{Z}^{n}$. We may suppose that the action on $X \subset V=\mathbb{C}^{N}$ is diagonal, and that there is no fixed point $(\neq 0)$ :

$$
g z=\left(e^{2 i \pi \mu_{k}(\gamma)} z_{k}\right) \quad \text { if } g=\gamma \bmod \mathbb{Z}^{n}
$$

where the $\mu_{k}(1 \leq k \leq N)$ are integral linear forms $\left(\mu_{k} \in \mathbb{Z}^{n} \subset \mathfrak{g}^{*} \simeq \mathbb{R}^{n}\right)$.

\footnotetext{
${ }^{10}$ Or reduced to the origin if we extend it as a closed subset of $\mathbb{C}^{N}$.
} 
Lemma 8. The action of $G$ is elliptic iff the characters $\mu_{k}$ generate a strictly convex cone in $\mathfrak{g}^{*}$.

Indeed, strict convexity is equivalent to the fact that $\sum \lambda_{k} \mu_{k}=0, \lambda_{k} \geq 0$ $\left(\lambda_{k}=z_{k} \bar{z}_{k}\right)$ implies $\lambda_{k}=0$ for all $k$.

The K-theoretic element defined by the Koszul (or de Rham) complex is

$$
\left[k_{V}\right]=\prod\left(1-\mu_{k}\right)
$$

and we get

$$
\operatorname{Ind}_{G}\left(\mathbb{H}_{V}\right)=\left[k_{V}\right]^{-1}=\prod\left(1-\mu_{k}\right)^{-1}
$$

where each factor $\left(1-\mu_{k}\right)^{-1}$ is expanded in positive powers of the $\mu_{k}$ (this is well defined if the $\mu_{k}$ generate a strictly convex cone); $\operatorname{Ind}_{G}\left(\mathbb{H}_{V}\right)$ is the partition function, given by a Hilbert-Samuel series. The $G$-index or asymptotic $G$-index of any equivariant Toeplitz system is then a multiple of $\chi\left(\mathbb{H}_{V}\right)$ in $\widehat{R}_{G}$ (resp. $\widehat{R}_{G}$ $\bmod R_{G}$ ).

The map $K^{G}(\mathbb{B}) \rightarrow K^{G}(X)$ always induces an isomorphism $R_{G} / R_{G} \cdot\left[k_{V}\right] \rightarrow$ $K^{G}(X)$ (for any action of $G$ ), so in the elliptic case, the equivariant asymptotic index is an isomorphism from $K^{G}(X)$ to $R_{G} \cdot\left[k_{V}\right]^{-1} / R_{G}$.

In the general case ( $G$ still a torus, but not an elliptic action), there may be several invariant elliptic subspheres $X_{J}=X \cap V_{J}$, corresponding to the subsets $J \subset[1, N]$ such that the $\mu_{j}, j \in J$, generate a strictly convex cone (i.e. there exists $v \in \mathfrak{g}$ such that $\mu_{j}(v)>0$ for $\left.j \in J\right)$. Each of them defines a Bott map $K^{G}\left(X_{J}\right) \rightarrow K^{G}(X-Z)$; an element of $K^{G}(X-Z)$ is in the range of this Bott map iff it is supported by $X_{J}$ (i.e. can be defined by an equivariant symbol $u$ invertible outside of $X_{j}$ ); the corresponding $G$-indices are the multiples of the inverse of the Koszul symbol $\left[k_{V_{J}}\right]^{-1}\left(\bmod R_{G}\right)\left(\left[k_{V_{J}}\right]=\left[d_{V_{J}}^{\prime \prime}\right]\right.$ with the notations above; and $\left[k_{V_{J}}\right]^{-1}$ should be expanded as a power series of the $\mu_{j}, j \in J$, as above).

Conjecture. Any element of $K^{G}(X-Z)$ is a sum of elements supported by elliptic subspheres $X_{J}$. In other words $K^{G}(X-Z)$ is generated by the K-theoretic elliptic elements $\left[k_{V_{J}}\right]$ associated to invariant elliptic subspheres (equivalently: by the images of the corresponding Bott maps).

Accordingly asymptotic indices are of the form $\sum P_{J}\left[k_{V_{J}}\right]^{-1}$, where $\left[k_{V_{J}}\right]$ denotes the K-theoretical element corresponding to the Koszul complex of elliptic subspheres (the inverse is understood as a series as above), and the $P_{J}$ are (finite) elements of $R_{G}$. More precisely: the asymptotic index map should be an isomorphism of $K^{G}(X-Z)$ to the $R_{G}$-submodule of $\widehat{R}_{G} / R_{G}$ generated by the $\mathbb{H}_{V_{J}}=\left[k_{V_{J}}\right]^{-1}$ for elliptic $V_{J} \subset V$. 
The assertion is easily seen to be true if $G=U(1)$ is the circle group $(n=1)$ : in this case $Z$ is a hypersurface of real codimension 1 ; it cuts $K^{G}(X-Z)$ (just as $X$ ) in two components, each of which is supported by a maximal positive (resp. negative) subsphere. The corresponding indices are those of the form

$$
P\left(\tau^{ \pm 1}\right)\left(k_{ \pm}\right)^{-1}
$$

where $\tau$ denotes the generator of $R_{G}$ (the tautological representation), $P$ is a polynomial, and

$$
k_{ \pm}=\prod_{ \pm n_{k}>0}\left(1-\tau^{n_{k}}\right)
$$

where the $n_{k}$ are the exponents of the action of $G=U(1)\left(e^{2 i \pi \theta} \cdot z=\left(e^{2 i \pi n_{k} \theta} z_{k}\right)\right)$; as above the inverse is expanded as a series of positive powers of $\tau^{ \pm 1}$.

The conjecture is also true ( $G$ a torus) if $Z$ is reduced to one 'interior' $N$ torus, i.e. the equations $\sum \lambda_{k} \mu_{k}=0$ have exactly one solution $\lambda_{k}>0$ up to a constant factor; equivalently: all hyperplanes $z_{k}=0$ are elliptic. In that case $G$ acts through an $N$-1-dimensional torus, and $Z / G$ is a one-dimensional circle. Let us first examine the case of an elliptic symbol $u: E \rightarrow F$ where $E, F$ are of rank one: then $u$ can be deformed into a monomial $z^{\alpha}$ (the characters of $E, F$ being linked by $\chi_{E}-\chi_{F}=\sum \alpha_{j} \chi_{k}$ ). Since a composition $v \circ v^{\prime}$ is stably homotopic to $v \oplus v^{\prime}, u$ is stably homotopic to a $\oplus u_{k}$ where $u_{k}$ is multiplication by one coordinate power $z_{k}^{\alpha_{k}}$ (resp. $\bar{z}_{k}^{-\alpha_{k}}$ if $\alpha_{k}<0$ ), and the corresponding element [uk] is supported by the subsphere $\left(z_{k}=0\right)$.

In the general case, because the quotient $Z / G$ is a circle, one sees easily that any equivariant homomorphism $u: E \rightarrow F$ can be deformed into a sum $\oplus u_{\alpha}: X \times E_{\alpha} \rightarrow X \times F_{\alpha}$ where the $E_{\alpha}, F_{\alpha}$ are representations of degree 1 , and our assertion follows.

The conjecture is finally also true if the set of characters $\mu_{k}$ is symmetric, i.e. $[1, N]$ is a union of disjoint pairs $\left(k, k^{\prime}\right)$ with $\mu_{k^{\prime}}=-\mu_{k}$. In this case it is not hard to see that our index situation can be deformed to the spherical case studied by M. F. Atiyah in 2. The 'maximal complex structures' of Atiyah, which provide the $\mathrm{K}$-theoretical generators, exactly correspond to the maximal elliptic subspheres. For a very elegant analysis of this, see also [10, 11]. Since any $G$-sphere can be embedded in a symmetric one, this at least shows that any index is a sum of indices supported by some convex cone (coming from some possibly larger elliptic sphere).

\section{References}

[1] M. F. Atiyah, K-theory, W. A. Benjamin, New York, 1967. Zbl 0159.53302 MR 0224083 
[2] _ Elliptic operators and compact groups, Lecture Notes in Math. 401, Springer, Berlin, 1974. Zbl 0297.58009 MR 0482866

[3] L. Boutet de Monvel, On the index of Toeplitz operators of several complex variables, Invent. Math. 50 (1979), 249-272. Zbl 0398.47018 MR 0520928

[4] Symplectic cones and Toeplitz operators, in Multidimensional complex analysis and partial differential equations (São Carlos, 1995, in honor of F. Trèves), Contemp Math. 205, Amer. Math. Soc., 1997, 15-24. Zbl 0896.58063 MR 1447211

[5] _ Vanishing of the logarithmic trace of generalized Szegö projectors, in Algebraic analysis of differential equations (in honor of T. Kawai), Springer, 2007, 67-78. Zbl 1142.32017

[6] _ Asymptotic equivariant index of Toeplitz operators, in Differential equations and exact WKB analysis, RIMS Kokyuroku Bessatsu B10, RIMS, 2008, 33-45. Zbl 1179.58013 MR 2498072

[7] L. Boutet de Monvel and V. Guillemin, The spectral theory of Toeplitz operators, Ann. of Math. Stud. 99, Princeton Univ. Press, 1981. Zbl 0469.47021 MR 0620794

[8] L. Boutet de Monvel, E. Leichtnam, X. Tang and A. Weinstein, Asymptotic equivariant index of Toeplitz operators and relative index of CR structures, in Geometric aspects of analysis and mechanics (in honor of H. Duistermaat), Progr. Math., Birkhäuser, to appear; arXiv:0808.1365v1.

[9] L. Boutet de Monvel and J. Sjöstrand, Sur la singularité des noyaux de Bergman et de Szegö, Astérisque 34-35 (1976), 123-164. Zbl 0344.32010 MR 0590106

[10] C. De Concini, C. Procesi and M. Vergne, Vector partition functions and generalized Dahmen-Michelli spaces, arXiv:0805.2907v2.

[11] , Vector partition functions and index of transversally elliptic operators, arXiv:0808.2545v1.

[12] L. Hörmander, Fourier integral operators I, Acta Math. 127 (1971), 79-183. Zbl 0212.46601 MR 0388463

[13] A. Melin and J. Sjöstrand, Fourier integral operators with complex valued phase functions, in Fourier integral operators and partial differential equations (Nice, 1974), Lecture Notes in Math. 459, Springer, 1975, 120-223. Zbl 0306.42007 MR 0431289

[14] _ Fourier integral operators with complex phase functions and parametrix for an interior boundary value problem, Comm. Partial Differential Equations 1 (1976), 313-400. Zbl 0364.35049 MR 0455054

[15] A. Weinstein, Some questions about the index of quantized contact transformations, in Geometric methods in asymptotic analysis, RIMS Kokyuroku 1014, 1997, 1-14. Zbl 0943.58019 MR 1625440 